\title{
Sozialpsychiatrie: die Geschichte eines Begriffs
}

Von Michael Shepherd

Wie Karl Jaspers begann Jean Starobinski seine berufliche Laufbahn als befähigter Arzt und praktischer Psychiater. Diese Ausbildung hat einen großen Teil seiner Arbeiten geprägt. Es sei in diesem Zusammenhang an eine Stelle in Jaspers' Werk «Allgemeine Psychopathologie» erinnert:

«Solcher Kenntnis sozialer Kreise bedarf der Psychiater immer zum Verständnis der ihm in der Klinik begegnenden Kranken. Darüber hinaus aber gewinnt die Psychopathologie zunehmend mehr Interesse für die abnormen seelischen Erscheinungen, die selten oder gar nicht innerhalb der Klinik studiert werden können. Sie erweitert ihre Erfahrungsgrundlage, indem sie Kenntnis zu gewinnen sucht von den abnormen Seelenvorgängen, die außerhalb der Klinik im freien Leben in den verschiedenen Kreisen menschlicher Gesellschaft vorkommen, und die die menschliche Geschichte ihr zeigt ... Die psychopathologische Wissenschaft beschränkt sich aber schon nicht mehr auf das Erfahrungsmaterial der Anstalten, sondern sucht in dem von der Vergangenheit überlieferten Material und in den seelischen Erscheinungen, die die Gegenwart außerhalb der Anstalten bietet, nach Erfahrungen, die sie innerhalb der Anstaltsmauern nicht machen kann.» ${ }^{1}$

Von der Formulierung Jaspers' sollte es nur ein kleiner Schritt zur Sozialpsychiatrie sein. Der Begriff wird zwar heute viel gebraucht; er bleibt aber umstritten und ist nicht eindeutig festgelegt. Die Unklarheit ergibt sich zum Teil aus der Vieldeutigkeit des Wortes «sozial». Zum Gebrauch und Mißbrauch des Wortes hatte schon vor 70 Jahren Grotjahn bemerkt:

«Leider wird gegenwärtig dieses Adjectivum häufig gerade von Ärzten und Vertretern der privaten Wohlfahrtspflege auch an unrechter Stelle angewandt. So heißt beispielsweise «sozial» niemals schlechthin nützlich und wirtschaftlich, ohne das Prädikat «sozial» zu verdienen. Das Wort ist auch nicht gleichbedeutend mit swohltätig für die unteren Bevölkerungsschichten〉, denn das könnte auch eine Volksküche oder eine Poliklinik sein, die kein Soziologe als soziale Einrichtung bezeichnen würde. Auch die «soziale Betätigung) in einer 〈sozialen Frauenschules ist zwar lobenswert, würde aber ihrer Bedeutung besser entsprechen, wenn sie in einer «Frauenschule für Wohlfahrtspflege` gelehrt würde. Gar der (Sozialarzt) ist eine bedauerliche Sprachbildung, die den schlichten Fürsorgearzt hoffentlich niemals verdrängen wird. Angesichts solchen sprachlichen Mißbrauches muß man wirklich noch daran erinnern, daß das Adjectivum social von dem lateinischen Wort socius ableitet und stets eine gesellschaftliche, gemeinschaftliche oder genossenschaftliche Beziehung voraussetzt». ${ }^{2}$

Wenn man behauptet, wie es ein Professor der Sozialpsychiatrie kürzlich getan hat, daß sein Fach «ein Teil der Sozialmedizin» ${ }^{3}$ sei, wird dadurch das 
Problem einer Begriffsdefinition nur verlagert, denn auch der Ausdruck «Sozialmedizin» ist unter Ärzten sehr umstritten, seit er von Guérin im Jahre 1848 geprägt und von Virchow im selben Jahr in Deutschland eingeführt wurde.

Jene Zeit der Umwälzung in Europa hat die Medizin, wie fast alle andern Gebiete menschlicher Aktivität, stark beeinflußt. Virchow hat den Zeitgeist sehr präzise ausgedrückt, als er in seiner Zeitschrift «Medizinische Reform» schrieb: «die Ärzte sind die natürlichen Anwälte der Armen und die soziale Frage fällt zum größten Teil in ihre Jurisdiktion».

Diese Feststellung ist ein Hinweis auf eine der beiden Hauptwurzeln der Sozialmedizin, nämlich die der humanitären Tradition in der Medizin. Auf eine klare Darstellung der zweiten Wurzel, nämlich des wissenschaftlichen Ursprungs dieser Disziplin, mußte man noch 60 Jahre lang warten, bis Grotjahn folgende sechs Grundsätze aufstellte:

1. Prävalenz: Die Bedeutung der Krankheit vom sozialen Gesichtspunkt aus wird in erster Linie bestimmt durch ihre Häufigkeit ... Die medizinische Statistik ist demnach die Grundlage jeder sozialpathologischen Betrachtung, da sie die krankhaften Erscheinungen der Menschen sowohl nach deren biologischen Verschiedenheit wie Alter, Geschlecht usw. als nach ihrer sozialen Differenzierung ermittelt.

2. Klinische Charakteristik: Eine Krankheit erhält noch nicht allein durch ihre Häufigkeit soziale Bedeutung ... Es erhebt sich also zweitens die Frage nach der Form, in der die betreffende Krankheit am häufigsten vorkommt ... Aber die charakteristische Form der klinischen Pathologie, der Schulfall, ist in der Regel nicht die Form, in der die Krankheit am häufigsten vorkommt oder am meisten durch soziale Verhältnisse bedingt ist oder letztere selbst wieder bedingt. Es ist daher durchaus erforderlich, neben dem klinischen und pathologisch-anatomischen Krankheitsbilde bei allen pathologischen Zuständen, die einer Untersuchung auf ihre Beziehungen zum Gesellschaftsleben überhaupt wert erscheinen, auch ihre sozialpathologisch typische Form festzustellen.

3. Die wichtigsten Beziehungen zwischen krankhaften Zuständen und den sozialen Verhältnissen liegen natürlich drittens auf ursächlichem Gebiete ... Am zweckmäßigsten hält sich deshalb die ursächliche Betrachtung an folgendes Schema: 1. Die sozialen Verhältnisse schaffen oder begünstigen die Krankheitsanlage. 2. Die sozialen Verhältnisse sind die Träger der Krankheitsbedingungen. 3. Die sozialen Verhältnisse beeinflussen den Krankheitsverlauf. 
4. Nicht nur werden krankhafte Zustände durch soziale Verhältnisse in Entstehung und Verlauf bedingt, sondern sie beeinflussen auch ihrerseits wieder die sozialen Zustände, besonders Bevölkerungsbewegung, Wehrkraft und Arbeitsleistung... Besonders durch ihren Ausgang wirken die Krankheiten auf die gesellschaftlichen Zustände ein. Dieser Ausgang kann bestehen in: 1.Tod. 2. Heilung. 3. Verkümmerung. 4. Siechtum. 5 . Veranlagung für andere krankhafte Zustände, und endlich 6 . in Entartung, d.h. in Verursachung einer Minderwertigkeit, die sich auf die Nachkommen vererbt.

5. Sind die Krankheiten für das Gesellschaftsleben von Bedeutung, so wird es auch ihre Beseitigung durch ärztliche Betätigung sein, soweit diese überhaupt möglich ist. Ein fünfter Punkt unserer Betrachtung wird daher sein, ob bei einer sozialpathologisch wichtigen Krankheit die ärztliche Behandlung überhaupt wesentliche Erfolge aufzuweisen hat und in welchem Maße sie die Bedeutung der Krankheit im sozialen Leben zu verändern imstande ist.

6. Wie können wir krankhafte Zustände durch soziale Maßnahmen in ihrem Verlaufe beeinflussen oder verhüten? Die Beantwortung dieser Frage führt die sozialpathologische Erörterung zu den Maßnahmen der sozialen Hygiene, deren Aufgabe, wie bereits erörtert, in der Verallgemeinerung der hygienischen Kultur und ihrer Ausdehnung auf die Gesamtheit der Bevölkerung ist.

Heute würde man diese Grundsätze als Gegenstand epidemiologischer Forschung betrachten, einer Forschungsrichtung, die sich mit den Krankheiten oder mit den medizinischen Bedürfnissen der Gesellschaft beschäftigt.

Die Entwicklung der Sozialpsychiatrie, die von Southard im Jahre 1917 als eine «Zwitterwissenschaft» eingeführt wurde, kann parallel dazu gesehen werden ${ }^{4}$. Vom achtzehnten Jahrhundert an läßt sich auch hier eine humanitäre Strömung erkennen und zwar in den Versuchen, einen Zusammenhang zwischen Geisteskrankheit und sozialen Faktoren wie Krieg, Industrialisierung, Armut und Überzivilisation aufzuzeigen, mit dem Ziel, die Lebensbedingungen durch soziale Reformen zu verbessern. George Rosen hat darauf hingewiesen, daß eine angemssene Analyse diese Prozesses sowohl unter dem Aspekt der Wissenssoziologie und der Psychiatriegeschichte erfolgen muß, als auch unter Anerkennung jener Geisteshaltung, derzufolge Sozialpsychiatrie ein Instrument des «human engineering» ist ${ }^{5}$. In der Auffassung eines der berühmtesten zeitgenössischen Vertreters dieser Position bezeichnet 
dieser Begriff zum Beispiel ein dehnbares Konzept, das alle sozialen, biologischen, pädagogischen und philosophischen Überlegungen einschließt, die die Psychiatrie unterstützen in ihrem Bemühen um größeren gesellschaftlichen Ausgleich und damit um die Verminderung psychiatrischer Notfälle ${ }^{6}$.

Den medizinischen Vertretern dieser Art von Sozialpsychiatrie haben die extremen Richtungen psycho-dynamischer Spekulation seit den Zwanzigerjahren dieses Jahrhunderts eine theoretische Grundlage geliefert. Insbesondere die Einführung der Gruppenpsychotherapie zur Zeit des Zweiten Weltkrieges führte zur nebulösen aber eindrucksvollen Idee der «therapeutischen Gemeinschaft», die auf gesellschaftliche Gruppen aller Art, von der Familie bis zu den Institutionen und zur Gesellschaft im allgemeinen, ihre Anwendung fand ${ }^{7}$. Der globale Einfluß dieses Konzepts spiegelt sich in den Aktivitäten der Weltgesundheitsorganisation wider: im Jahre 1959 ging ein Expertenkomitee für seelische Gesundheit sogar so weit, die Sozialpsychiatrie zu definieren als «diejenigen präventiven und kurativen Maßnahmen, die es dem Individuum ermöglichen, ein befriedigendes und nützliches Leben im Rahmen seiner Umwelt zu führen». ${ }^{8}$

Soviel zum humanitären Ansatz in der Sozialpsychiatrie. Die wissenschaftliche Beschäftigung mit den sozialen Aspekten der Psychiatrie kann unabhängig davon in der Arbeit von Ärzten des 19. und frühen 20. Jahrhunderts verfolgt werden. Vor etwas über hundert Jahren schrieb Henry Maudsley: «Es schien gerechtfertigt notwendig zu betonen, daß Geisteskrankheit in Wirklichkeit ein gesellschaftliches Phänomen ist, und darauf zu bestehen, daß sie nicht zufriedenstellend erforscht und verstanden werden kann, wenn sie nicht aus einem sozialen Blickwinkel gesehen wird». ${ }^{9} \mathrm{Zu}$ Beginn dieses Jahrhunderts wurde die Richtigkeit dieser Beobachtung in Frankreich und besonders auch in Deutschland erkannt, wie es in Aschaffenburgs im Jahre 1915 veröffentlichtem «Handbuch» und in den Arbeiten von Wilmanns, Kronfeld und Birnbaum dargestellt ist. Die vollständige Entwicklung dieses Gedankens sollte jedoch von der systematischen Anwendung epidemiologischer Methoden abhängen. Hierin besteht der besondere Beitrag angelsächsischer Forscher ${ }^{10}$. Goldberger mit seiner Arbeit über Pellagra ${ }^{11}$ und Gajdusek mit seiner Studie über die Kurukrankheit ${ }^{12}$ bieten vielleicht die besten Beispiele einer Anwendung dieses Forschungsansatzes bei einzelnen Krankheiten. Der britische Psychiater Sir Aubrey Lewis, der die allererste Forschungsabteilung ausschließlich für Sozialpsychiatrie gründete ${ }^{13}$, hat die besondere Tragweite dieses Ansatzes wohl am deutlich- 
sten gesehen. Es ist aber vielleicht von Bedeutung, daß diese Abteilung schon 10 Jahre existierte, bevor Lewis, sich der Vagheit des Konzeptes vollkommen bewusst, den Begriff «Sozialpsychiatrie» als einen Ausdruck anerkannte, der seinem Forschungsprogramm die notwendige wissenschaftliche Anerkennung verleihen würde.

Lewis' Konzept der Sozialpsychiatrie basiert auf drei Grundpfeilern: erstens auf der Anwendung epidemiologischer Prinzipien auf Forschungsprojekte, wobei Gesellschaft, Klinik und Labor als Informationsquelle und Experimentierfeld dienen. Zweitens wollte er - soweit möglich - die sozialen Aspekte bestehender klinischer Konzepte identifizieren, um sie genau zu erforschen; so wurde aus «Rehabilitation» «Behandlung unter sozialen Gesichtpunkten» und "Behinderungen» wurde umdefiniert in «messbare Symptome, die für das Sozialverhalten von Bedeutung sind». Drittens betonte er die Notwendigkeit, die Methoden und Konstrukte der Sozialwissenschaften zu nutzen, ohne dabei deren Grenzen außer acht zu lassen. In Lewis' eigenen Worten heißt es:

«... der soziale Aspekt der Psychiatrie ist derart durchdringend und ist es in der Geschichte der Psychiatrie auch immer gewesen, daß ich nicht glauben kann, er hätte jemals so vernachlässigt werden können, wenn die Forschungsmethoden besser entwickelt gewesen wären. Insbesondere das Gebiet der Soziologie befindet sich noch im Entwicklungsstadium.»

Diese Sicht ist natürlich von den Soziologen, insbesondere in den Vereinigten Staaten, nicht geteilt worden. Nach einer Zeit steigenden Interesses in den zwanziger Jahren dieses Jahrhunderts ging Brown im Jahre 1934 sogar so weit zu behaupten, daß das Gebiet der Sozialpsychiatrie alle abnormen Formen sozialer Anpassung, sowohl von Individuen als auch von Gruppen, umfasse. Wie die Sozialpsychologie sei die Sozialpsychiatrie ein Teilbereich der Soziologie ${ }^{14}$. Und noch 1948 nannte Warren Dunham das Fach «eine Schöpfung der Soziologen», um einen Namen zu finden für die Interessen einiger ihrer Berufsgenossen, die über Persönlichkeitsstörungen forschen ${ }^{15}$. Obwohl dieser Versuch, Sozialpsychiatrie als eine Mischung aus Kulturanthropologie, Theorie interindividueller Beziehungen und psychoanalytischer Spekulation zu integrieren, sich als unfruchtbar erwies, haben verschiedene Wissenschaftler seit Durkheims früher Studie über Selbstmord die epidemiologischen Methoden berechtigterweise angewandt. Dunhams eigene Studie über die Ökologie der Schizophrenie in Chicago ist ein Musterbeispiel seiner Art. Wenn auch epidemiologische Forschung, wie ein berühmter Medizinsoziologe feststellte, eine Art von Forschung ist, mit der 
er und seine Kollegen sich ihr ganzes Berufsleben hindurch beschäftigt haben ${ }^{16}$, so bdeutet das allein noch nicht, daß die Sozialwissenschaften sich die Sozialpsychiatrie einfach einverleiben können.

Glücklicherweise hat sich der bloße Meinungsstreit zu einer konstruktiven Diskussion entwickelt. Mittlerweile liegt genug Forschungsarbeit vor, um zu zeigen, daß Grotjahns sechs Kategorien, nämlich Häufigkeit, Form, Ursprung, Prognose, Therapie und Verhütung von Geisteskrankheiten, wissenschaftlich untersucht werden können. Außerdem wird die Bedeutung dieser Arbeit, gleichgültig ob sie von einem medizinischen oder soziologischen Untersucher durchgeführt worden ist, von den in Verwaltung, Klinik und Forschung Tätigen gleichermaßen anerkannt. Auf diese Weise bereichert sie das Gebiet der allgemeinen Psychopathologie, in deren Rahmen die Sozialpsychiatrie ihren unentbehrlichen Platz einnimmt und wozu Jean Starobinski einen denkwürdigen Beitrag geleistet hat.

\section{Bibliographie}

${ }^{1}$ Jaspers, K. (1973): Allgemeine Psychopathologie: 9. Auflage. Springer Verlag, Berlin.

${ }^{2}$ Grotjahn, A. (1923): Soziale Pathologie. 3. Auflage. Berlin: Springer.

${ }^{3}$ Wing, J. K. (1980): Innovations in social psychiatry. Psychological Medicine, 10, 219-230.

${ }^{4}$ Southard, E. E. (1917): Alienists and Psychiatrists. Mental Hygiene, 1, 567-571.

5 Rosen, G. (1959): Social stress and mental disease from the 18th century to the present: some origins of social psychiatry. The Milbank Memorial Fund Quarterly, XXXVII, No. 1, 5-32.

${ }^{6}$ Jones, M. (1968: Social Psychiatry in Practice. Penguin Books: Harmondsworth, p. 30

7 Hare, E. H. (1969): The relation between social psychiatry and psychotherapy. In «Psychiatry in a Changing Society), ed. S. H. Foulkes \& G.S. Prince, pp. 3-16, Tavistock: London.

${ }^{8}$ World Health Organization (1959): Social Psychiatry and Community Attitudes. Technical Report Series No.177. WHO: Geneva.

${ }^{9}$ Maudsley, H. (1879): The Pathology of Mind. Macmillan: London.

${ }^{10}$ Shepherd, M. (1975): Epidemiologische Psychiatrie, in Psychiatrie der Gegenwart, Band III, zweite Aufl. ed. K.P. Kisker, J.-E. Meyer, C. Müller \& E.Strömgren. Berlin: Springer, 119-149.

11 Goldberger, J. (1927): De Lamar Lectures. Williams \& Wilkins: Baltimore.

12 Gajdusek, D.C. \& Zigas, V. (1959): Clinical, pathological and epidemiological study of an acute progressive degenerative disease of the central nervous system among natives of the Eastern Highlands of New Guinea. American Journal of Medicine, 26, 442-469.

13 Shepherd, M. (1980): From social medicine to social psychiatry: the achievement of Sir Aubrey Lewis. Psychological Medicine, 10, 211-218.

14 Brown, L.G. (1934): (Social Psychiatry〉 in 〈The Field and Methods of Sociology) ed. L. L. Bernard, New York: Long \& Smith.

15 Dunham, H. W. (1948): Social psychiatry. American Sociological Review, 13, 183-187. 
${ }^{16}$ Mechanic, D. (1970): Problems and prospects in psychiatric epidemiology. In Psychiatric Epidemiology, eds. E. H. Hare \& J. K. Wing, Oxford University Press: London.

\title{
Summary
}

This paper traces the origins and directions of the concept of ssocial psychiatrys. The humanistic and scientific aspects of the term are distinguished. The heuristic value of the approach, it is suggested, depends on the methods of clinical epidemiology.

\author{
Michael Shepherd \\ Professor of Epidemiological Psychiatry \\ Institute of Psychiatry \\ University of London \\ De Crespigny Park \\ Denmark-Hill \\ London SE 5
}

